\title{
PROPERTIES OF FeCo NANOPOWDER PREPARED BY CHEMICAL SYNTHESIS
}

\author{
Karel Zábranský — Oldřich Schneeweiss *
}

\begin{abstract}
Chemical synthesis of binary Fe-Co oxalate was used for preparation of Fe-Co nanoparticles. X-ray difftaction spectra recorded during temperature treatment in reduction atmosphere show two main stages of transformation: amorphization of the oxalate mixture and formation of Fe-Co nanoparticles. After X-ray diffraction high temperature treatment the nanoparticles with $50 \mathrm{~nm}$ mean crystallite were found. Comparison of magnetic properties during and after different type of temperature treatment shows that coercivity and saturation magnetic polarization are sensitive in dependence on sample condition, length of treatment and temperature. Analysis of Mössbauer spectra reveal several components which were ascribed different phases during temperature treatments.
\end{abstract}

\section{INTRODUCTION}

Iron-cobalt nanoparticles are well known for their good magnetic properties. A high value of magnetization, low coercivity and high Curie temperature create a potential for applications in medicine, high density recording media or as a catalyst [1-3]. Several methods for preparation FeCo have been developed recently, eg chemical or physical vapor deposition, sol-gel technique, laser pyrolysis. Thermal decomposition of oxalate precursor was applied as well [4]. In our work the different procedure for preparation of binary Fe-Co oxalate was used, namely creation solid state mixture by segregation from solution allows mixing of the different metal species on an atomic scale. In the case of materials containing more than one metal, this method also allows a good control over the relative proportions of the elements. In this work we studied the process of synthesis the nanocrystalline phase in dependence on temperature treatment, and the properties of the prepared material.

\section{EXPERIMENTAL DETAILS}

The binary solid state Fe-Co oxalate precursor was prepared by segregation from Fe and Co sulfate solution. The nanoparticles were prepared by thermal decomposition of binary $\mathrm{Fe}-\mathrm{Co}$ oxalates in a hydrogen atmosphere.

The phase analysis of the samples was carried out by X-ray diffraction (XRD) using $\mathrm{CoK} \alpha$ radiation. High temperature XRD measurements were done in Anton Paar TCU 2000 furnace with a platinum heating strip. The high temperature XRD measurement was used for the study of the formation of FeCo nanocrystalline phase. After the short scan ( $2 \Theta$ from 38 to $66^{\circ}$ for 30 minutes) measurement at $100^{\circ} \mathrm{C}$ the temperature increased gradually to $600{ }^{\circ} \mathrm{C}$ with the step $\Delta T=20^{\circ} \mathrm{C}$ and for every step the short scan was taken. Finally the diffraction in the whole $2 \Theta$ range was measured at room temperature. The sample was for the whole time under hydrogen atmosphere. For better determination of the beginning of precursor amorphization and formation of $\mathrm{FeCo}$ phase the measurement with the temperature steps $\Delta T=5^{\circ} \mathrm{C}$ were taken in the critical temperature region $\left(150\right.$ to $\left.300^{\circ} \mathrm{C}\right)$.

Additional information on size morphology and structure was obtained using transmission electron microscopy.

Mössbauer spectroscopy (MS) was performed at room temperature and at several low temperatures. Spectra were taken in the transmission geometry using the ${ }^{57} \mathrm{Co} / \mathrm{Rh}$ source and evaluated using CONFIT program package [5]. Velocity scale was calibrated with a standard thin $\alpha$-iron foil.

Fe-Co oxalate precursor was annealed in resistance furnace at $560^{\circ} \mathrm{C}$ in hydrogen atmosphere for 2 hours.

Hysteresis loops were measured by vibrating sample magnetometer in magnetic field up to $796 \mathrm{kA} / \mathrm{m}$. Thermomagnetic curves were taken at constant field $4 \mathrm{kA} / \mathrm{m}$ in $\mathrm{H}_{2}$ atmosphere.

Temperature regime was $20 \rightarrow 800 \rightarrow 20{ }^{\circ} \mathrm{C}$ with the temperature sweep ${ }^{\circ} \mathrm{C} / \mathrm{min}$. Before and after the thermomagnetic measurements hysteresis loops were measured.

\section{RESULTS AND DISCUSSION}

X-ray diffraction patterns of the as-prepared precursorsample confirm the requested composition, Fe-Co oxalate. The recorded patterns show also traces of iron sulfate which indicates that impurities remained from the precursor preparation. In Fig. 1 selected high temperature XRD

* Institute of Physics of Materials, AS CR, Brno, Czech Republic, zabransky@ipm.cz 

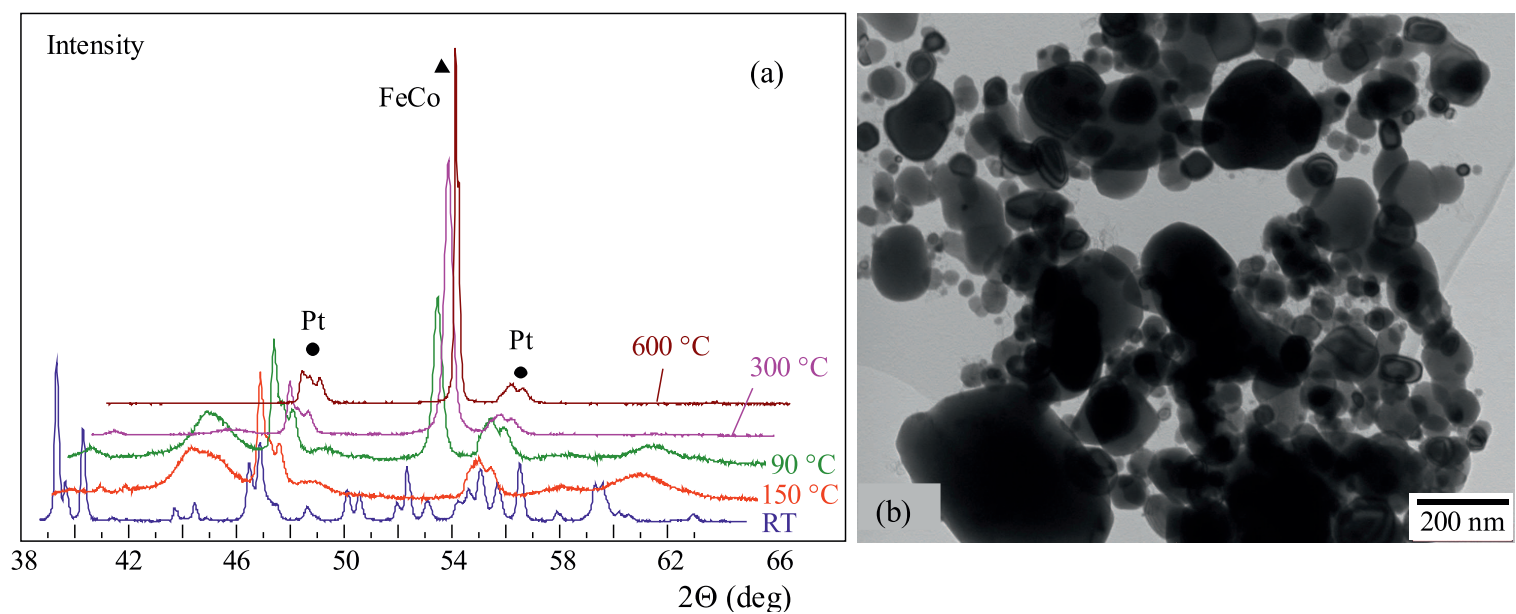

Fig. 1. XRD patterns measured at different annealing temperatures (a), TEM image of the nanoparticles (b)
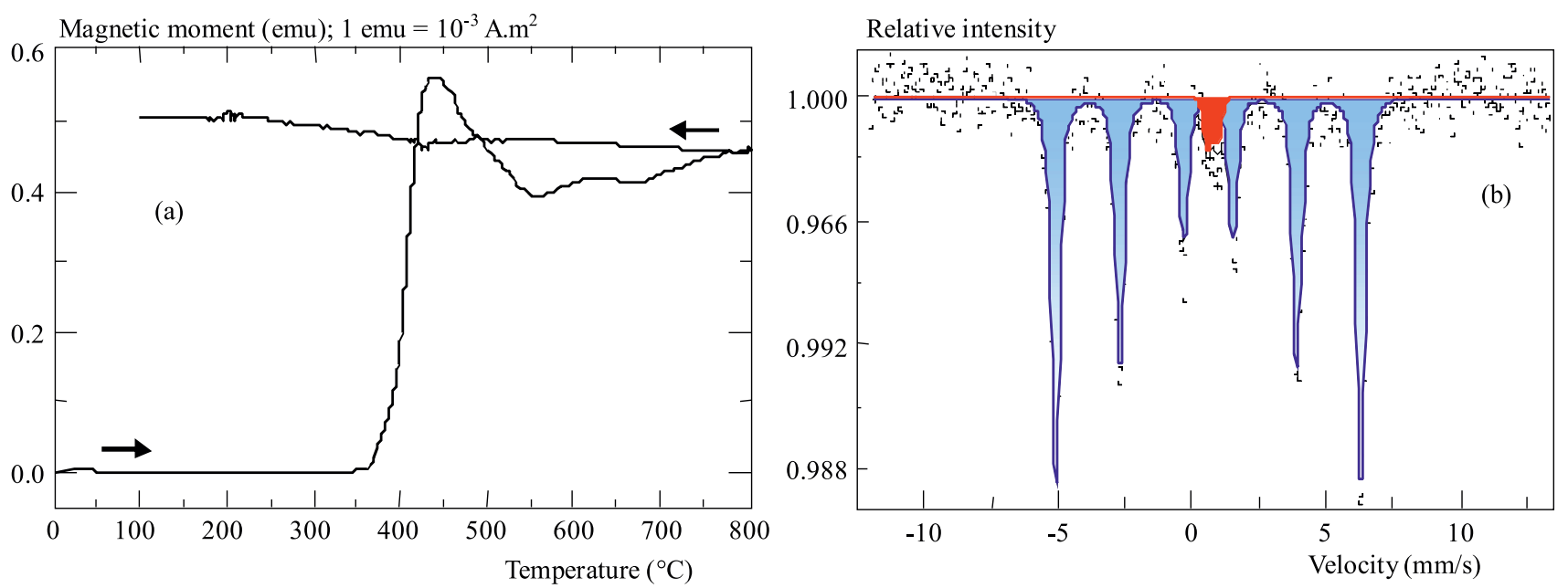

Fig. 2. Thermomagnetic curve of the Fe-Co oxalate taken in hydrogen atmosphere, dynamic (a), Mössbauer spectrum of the sample after the measurement of the thermomagnetic curve. The sextet components are filled by diagonal cross and the doublet by black (b)

Table 1. Parameters of the hysteresis loops taken after different heat treatments of the precursor in hydrogen atmosphere (coercivity, $H_{c}$, saturation polarization $J_{s}$, remanent polarization $J_{r}$ )

\begin{tabular}{lccc}
\hline Heat treatment & $H_{c}(\mathrm{kA} / \mathrm{m})$ & $J_{s}(\mathrm{~T})$ & $J_{r}(\mathrm{~T})$ \\
\hline After thermomagnetic curve up to $800{ }^{\circ} \mathrm{C}$ & 1.52 & 2.45 & 0.046 \\
After high temperature XRD up to $600{ }^{\circ} \mathrm{C}$ & 14.24 & 2.32 & 0.395 \\
After furnace annealing up to $560^{\circ} \mathrm{C}$ & 23.20 & 1.68 & 0.440 \\
\hline
\end{tabular}

Table 2. The hyperfine parameters of Mössbauer spectra: atomic fraction of iron atoms A, hyperfine field $B_{\mathrm{hf}}$, distribution of hyperfine field, $\Delta B_{\mathrm{hf}}$, isomer shift $\delta$, quadrupole shift $\varepsilon$, quadrupole splitting, $\Delta E_{\mathrm{Q}}$

\begin{tabular}{lcccccc}
\hline Component & $\mathrm{A}(\%)$ & $B P_{\mathrm{hf}}(\mathrm{T})$ & $\Delta B_{\mathrm{hf}}(\mathrm{T})$ & $\delta(\mathrm{mm} / \mathrm{s})$ & $\varepsilon(\mathrm{mm} / \mathrm{s})$ & $\Delta E_{\mathrm{Q}}(\mathrm{mm} / \mathrm{s})$ \\
\hline 1. Sextet & $78 \pm 2$ & $34.1 \pm 0.1$ & - & $0.03 \pm 0.01$ & $0.02 \pm 0.01$ & - \\
2. Sextet & $16 \pm 2$ & $35.0 \pm 0.1$ & $2.0 \pm 0.2$ & $0.02 \pm 0.01$ & $-0.93 \pm 0.02$ & - \\
Doublet & $6 \pm 1$ & - & - & $0.14 \pm 0.01$ & - & $0.49 \pm 0.01$ \\
\hline
\end{tabular}

patterns are shown. At $150^{\circ} \mathrm{C}$ the amorphization of precursor which is the first stage of decomposition of the FeCo oxalate was found. Solid solution $\alpha$-FeCo diffraction peaks appeared at $290^{\circ} \mathrm{C}$. The mean coherence length is increasing with rising temperature which is represented by peaks intensity and line widths. This is a consequence of ordering processes in the FeCo phase. Using the Scherrer equation the mean coherence length was calculated and it is increasing from $\sim 30 \mathrm{~nm}$ at $290{ }^{\circ} \mathrm{C}$ to $\sim 90 \mathrm{~nm}$ at the end of the heat treatment. The XRD patterns of the 
sample after temperature treatment and exposition to air atmosphere show only the FeCo phase without any traces of oxides. This good resistance against oxidation can be ascribed to the high content of cobalt in the nanopowder.

The image from transmission electron microscopy of the sample after the high temperature XRD measurement is shown in Fig. 1 a. A broad size distribution (approximately 40 to $200 \mathrm{~nm}$ ) can be observed there. EDX analysis of selected particles proved that the chemical composition ratio of Fe to Co is 50 : 50 with very low fluctuations. The point diffractions confirm the bcc structure of the particles.

The thermomagnetic curve shown in Fig. 2 exhibits a rapid increase in the temperature range 350 to $-400{ }^{\circ} \mathrm{C}$ which is due to formation of the FeCo ferromagnetic phase. The slow decrease above $400{ }^{\circ} \mathrm{C}$ is probably associated with magnetic hardening caused by grain growth and formation of magnetocrystalline anisotropy. The fine increase with decreasing temperature is in agreement with the temperature dependences of magnetic moments of ferromagnetic materials. The difference can be ascribed to different forms of the samples. While for the thermomagnetic measurement a compacted (pressed) form of a small cylinder was used, the powder for XRD measurements was strewn on the heating Pt strip. The access of hydrogen to the particles was much easier in case of XRD measurement, and therefore the chemical reduction is easier there.

The parameters derived from the hysteresis loops taken after different heat treatments are summarized in Table 1. The differences in all parameters can be ascribed to the grain size and free surface area of the particles during the heat treatment. Coercivity $H_{c}$ is decreasing with an increase of the maximum temperature of annealing, ie with grain size. On the contrary, saturation polarization $J_{s}$ is increasing and it corresponds to the increasing ratio of atoms in grain interiors to grain surfaces.

The Mössbauer spectrum of the sample which was treated during thermomagnetic curve measurement is drawn in Fig. 2 b. Three components were used for good fit of the experimental data: (i) the sextet with the line width close to the line width obtained for the sextet in the spectrum of iron in the calibration sample, (ii) broad sextet described by distribution of hyperfine field, (iii) the doublet. Parameters of the components are given in Table 2. The Mssbauer spectrum of the sample after high temperature XRD yielded the same results. The high value of hyperfine field of the first sextet implies that it represents FeCo nanoparticles. The second sextet described by the distribution of hyperfine field corresponds to iron atoms in surface regions of the nanoparticles and possible fluctuations in chemical composition. The doublet can be ascribed to superparamagnetic particles. Ac- cording to [6] the superparamagnetic state is achieved for particles FeCo smaller than $34 \mathrm{~nm}$. Furthermore, in the spectrum taken on the sample after the thermomagnetic curve measured to $560{ }^{\circ} \mathrm{C}$ only a higher atomic fraction of the doublet was analyzed.

\section{CONCLUSION}

We have shown that FeCo nanoparticles can be prepared by reduction of binary FeCo oxalate in hydrogen atmosphere. The chemical composition of the powder was close to the requested concentration $\mathrm{Fe}_{50} \mathrm{Co}_{50}$. XRD, Mssbauer spectroscopy and TEM results confirmed the bcc structure of the particles. Comparison of the critical temperatures for decomposition of the precursor and formation of FeCo particles was obtained from temperature dependences of XRD diffractions and magnetic moment measurements. They indicate that the kinetics of the process is strongly influenced by the form of the sample. A similarly large difference can be observed in the parameters of hysteresis loops taken after different methods of heat treatment.

\section{Acknowledgments}

This work was supported by the Ministry of Education, Youth and Sports of the Czech Republic (Project No. 1M6198959201) and by the Grant Agency of the Czech Republic (Project No. 106/08/1440).

\section{REFERENCES}

[1] A. FiguerolaA, A.-Di CORATOB, R.-MANNAA, L.PELlEGRINO, T. : Pharmacol. Res., in press (2010).

[2] KODAMA, R. H.: J. Magn. Magn. Mat. 200 (1999), 359-372.

[3] YUAN, P.-WU, H.-XU, H.-XU, D.-CAO, Y.-WEN WEI, X. : Mater. Chem. Phys. 105 (2007), 391-394.

[4] HORWITZ, H. S.-LONGO, J. M.: Mater. Res. Bull. 13 (1978), 1359-1369.

[5] ZAK, T.-JIRASKOVA, Y.: Surf. Interf. Anal. 38 (2006), 710-714.

[6] MAJTEJCH, S.-JIN, Y. : Science 284 (1999), 470.

Received 30 June 2010

Karel Zábranský, Oldřich Schneeweiss. Biographies not supplied.

$* * *$

This work was presented at Nanoved \& Nanotech \& Techtransfer 2010, International Conference on Nanoscience, Nanotechnology, Nanomaterials, Nanomedicine and Transfer 1619 May 2010, Bratislava, Slovakia. 